\title{
Heavy-flavour observables within a (3+1)D transport approach
}

\author{
Andrea Beraudo*, A. De Pace, M. Monteno, M. Nardi and F. Prino \\ INFN - Sezione di Torino \\ Via Pietro Giuria 1, I-10125 Torino \\ E-mail: beraudoeto.infn. it
}

\begin{abstract}
In this contribution we display our first predictions for heavy-flavour observables in heavy-ion collisions obtained extending our well-established POWLANG transport setup to perform full $(3+1) D$ simulations. In particular, we show our findings for the directed-flow coefficient $v_{1}$ and for the nuclear modification factor $R_{\mathrm{AA}}$ and elliptic-flow $v_{2}$ at forward rapidity: in both cases one needs to drop the approximation of longitudinal boost-invariance, usually enforced in most numerical implementations of the transport equations and assumed in our past publications. We briefly discuss how from a theory-to-data comparison one can get a richer $(3+1) \mathrm{D}$ information on the initial geometry of the fireball and put tighter constraints on the heavy-flavour transport coefficients.
\end{abstract}

HardProbes 2020

1-6 June 2020

Austin, Texas

\footnotetext{
* Speaker.
} 


\section{Introduction}

Heavy-flavour (HF) particles arising from parent charm and beauty quarks are among the best probes of the medium formed in relativistic heavy-ion collisions (HIC's). Due to their large mass heavy quarks (HQ's) are produced very early after the collision, before the formation of a thermalized Quark-Gluon Plasma (QGP). Hence, before hadronizing they cross the fireball produced in the collision probing all the stages of its evolution, performing a tomography of the latter. Furthermore, theoretical estimates indicate that the relaxation time of HQ's is of the same order as the lifetime of the QGP, so that one expects that charm and beauty approach only partial thermal equilibrium with the fireball through which they propagate: deviations from full thermalization observed in the data have thus the potential to put phenomenological constraints on the HQ transport coefficients.

So far most theoretical calculations developed for the study of HF observables are based on a $(2+1) \mathrm{D}$ modelling of the background medium, assuming its invariance for longitudinal boosts: this usually suffices for most experimental measurements, which are performed around mid-rapidity. Dropping such an approximation and considering a full $(3+1) \mathrm{D}$ hydrodynamic evolution of the fireball open the possibility of extending our transport predictions over a wider rapidity range and of addressing observables like the directed flow $v_{1}(y)$ of $D$ mesons, a promising measurement with the potential of providing a rich physical information on the matter produced in HIC's. This is the subject of our contribution.

\section{The (3+1)D POWLANG approach: general setup and results}
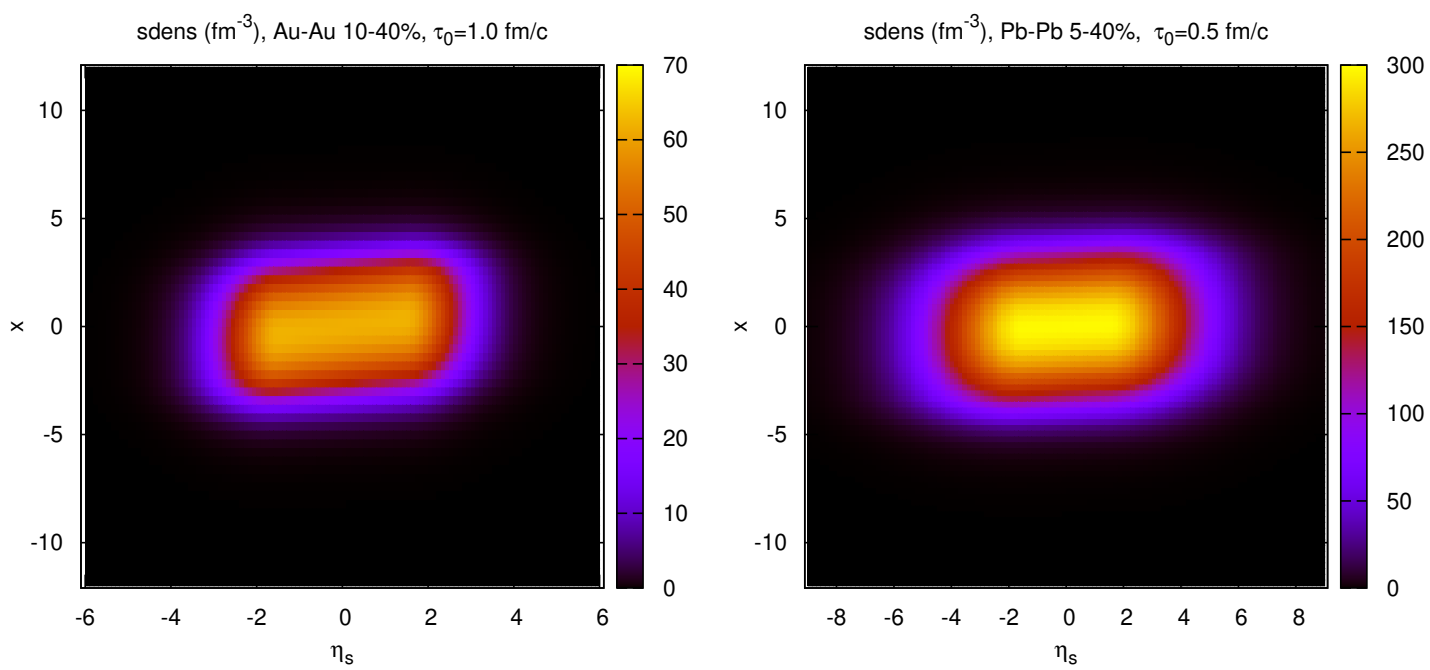

Figure 1: The initial entropy density in the $\eta_{s}-x$ plane at $y=0$ in Au-Au collisions at $\sqrt{s_{\mathrm{NN}}}=200 \mathrm{GeV}$ (left panel) and $\mathrm{Pb}-\mathrm{Pb}$ collisions at $\sqrt{s_{\mathrm{NN}}}=5.02 \mathrm{TeV}$ (right panel).

In order to set the initial conditions for our (3+1)D hydrodynamic equations, following Ref. [1], we employ the optical Glauber model, assuming that the initial entropy deposition arises from a linear combination of the contributions from the participant ("wounded") nucleons and from the binary nucleon-nucleon collisions. In particular right/left-moving wounded nucleons are assumed 
to produced more particles at forward/backward rapidity. The net effect is that at the initial time $\tau_{0}$ the fireball turns out to be tilted in the $\eta_{s}-x$ plane $\left(\eta_{s} \equiv(1 / 2) \ln [(t+z) /(t-z)]\right.$ being the spatial rapidity), as shown in Fig. 1. For deeper details we refer the reader to Ref. [1].
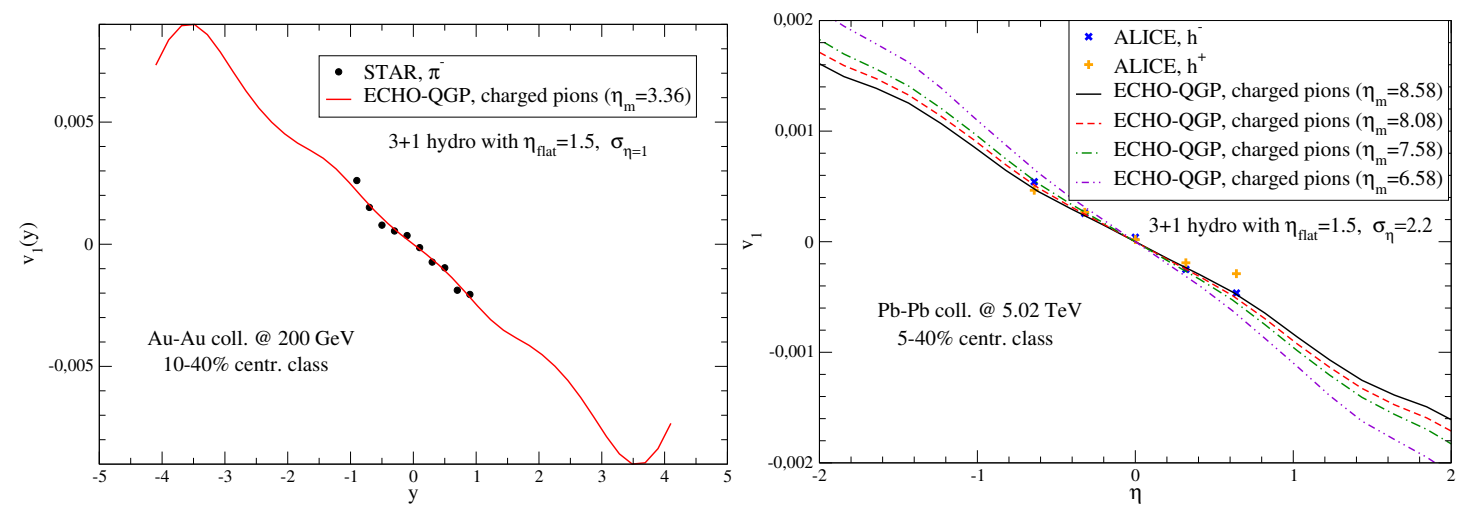

Figure 2: The directed flow $v_{1}(y)$ of charged pions in Au-Au collisions at $\sqrt{s_{\mathrm{NN}}}=200 \mathrm{GeV}$ (left panel) and $\mathrm{Pb}-\mathrm{Pb}$ collisions at $\sqrt{s_{\mathrm{NN}}}=5.02 \mathrm{TeV}$ (right panel). Results obtained with our hydrodynamic background are compared to experimental data obtained by the STAR [2] and ALICE [3] collaborations at RHIC and at the LHC, respectively. The coefficient $\eta_{m}$ controls the initial tilting of the fireball.

The hydrodynamic expansion maps the tilted geometry into a dipole asymmetry in the azimuthal emission of soft particles, quantified by the directed-flow coefficient $v_{1} \equiv\left\langle\cos \left(\phi-\psi_{\mathrm{RP}}\right)\right\rangle$. Before moving to HF transport calculations we wish to validate our hydrodynamic background, described the the ECHO-QGP code [4], against experimental data. Hence in Fig. 2 we compare our findings for the pion $v_{1}$ in non-central collisions to STAR [2] and ALICE [3] measurements.
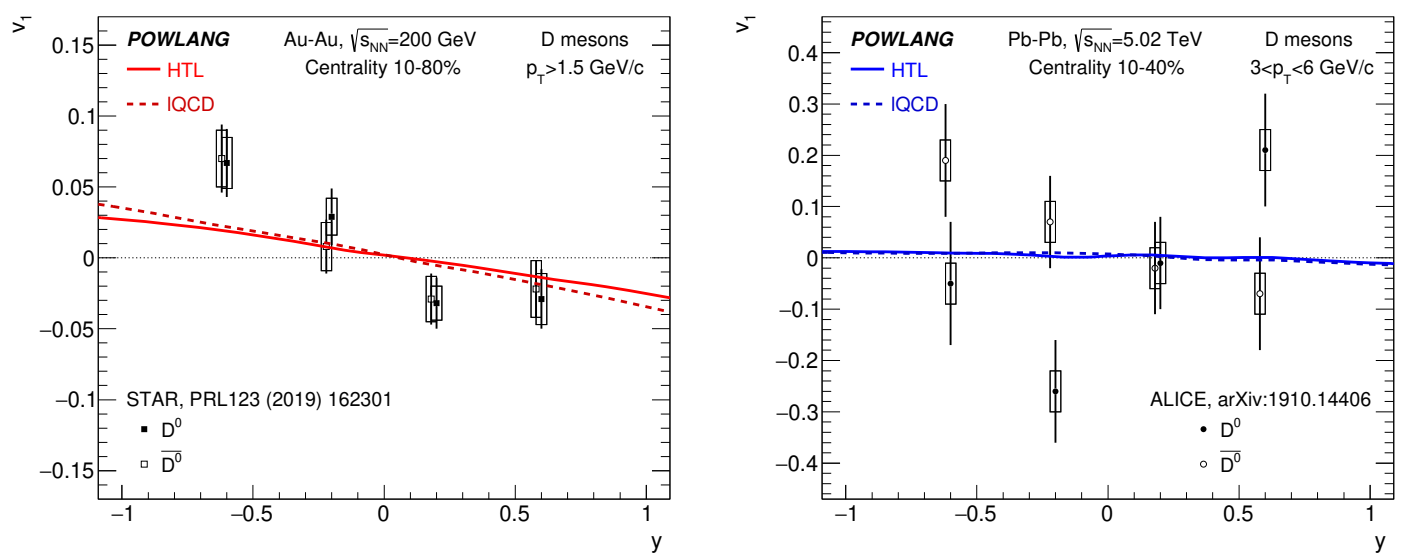

Figure 3: The directed flow $v_{1}(y)$ of $D$ mesons in Au-Au collisions at $\sqrt{s_{\mathrm{NN}}}=200 \mathrm{GeV}$ (left panel) and $\mathrm{Pb}-\mathrm{Pb}$ collisions at $\sqrt{s_{\mathrm{NN}}}=5.02 \mathrm{TeV}$ (right panel) as a function of the particle rapidity. POWLANG results for the average $D^{0}+\overline{D^{0}}$ flow with different different choices of transport coefficients are compared to experimental data for $D^{0}$ and $\overline{D^{0}}$ obtained by the STAR [5] and ALICE [3] collaborations.

We now address the issue of the propagation of HQ's in the tilted background above described. As discussed in detail in previous publications the transport of charm and beauty quarks in the QGP is simulated through a relativistic Langevin equation [6]. Results obtained with different transport 
coefficients are compared, arising either from a weak-coupling calculation with resummation of medium effects (HTL scheme) or from lattice-QCD simulations (IQCD scheme). We stress that, at variance with the fireball, the spatial distribution of HQ's at the initial thermalization time of the bulk medium $\tau_{0}$ does not display any distortion in the $\eta_{s}-x$ plane. Being produced in hard events, their initial location in the transverse plane simply follows from the $n_{\text {coll }}(x)$ distribution and their longitudinal coordinate $\eta_{s}$ coincides with the initial HQ rapidity $y$, assuming a free-streaming motion $z=v_{z} t$ before the formation of a thermalized medium. In the case of HF particles we have then a second important contribution to the $v_{1}$ coefficient beside the directed flow inherited from the fireball, namely the different medium thickness crossed by HQ's of a given rapidity moving along the positive/negative $x$-direction. This explains the much larger value of $v_{1}$ found for charm particles than for soft hadrons, as shown in Fig. 3, where we compare our predictions to STAR [5] and ALICE [3] data. Notice that experimental collaborations provide separate results for $D^{0}$ and $\overline{D^{0}}$ mesons. There are theoretical suggestions that possible differences between the two set of data can be attributed to the strong initial electromagnetic fields present in the fireball [7,8], however current experimental results do not allow one to draw definite conclusions.
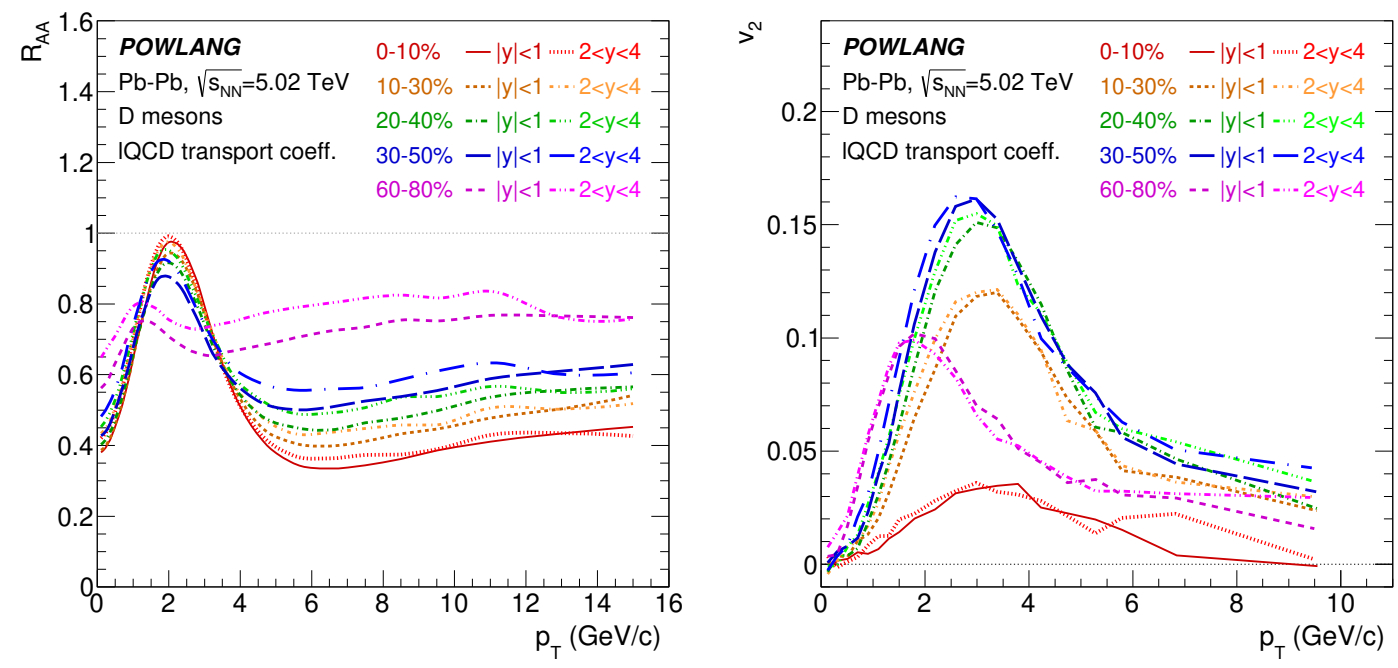

Figure 4: The nuclear modification factor $R_{\mathrm{AA}}$ (left panel) and elliptic-flow coefficient $v_{2}$ (right panel) of $D$ mesons in $\mathrm{Pb}-\mathrm{Pb}$ collisions at central and forward rapidity. Results for various centrality classes are displayed. Our findings, in particular in the case of $v_{2}$, display only a mild dependence on rapidity.

Finally, the full (3+1)D numerical solution of the hydrodynamic equations for the background allows us to extend our predictions for HF observables to forward rapidity. Hence in Fig. 4 we display POWLANG results (predictions with lattice-QCD transport coefficients are shown) for the nuclear modification factor $R_{\mathrm{AA}}$ and elliptic-flow coefficient $v_{2}$ of $D$ mesons in two different rapidity intervals. Differences between results at mid and forward rapidity are mild: negligible in the case of $v_{2}$, a bit larger for the $R_{\mathrm{AA}}$, compatible with the expectation that at forward rapidity the lower density of the medium should lead to a milder quenching of the momentum distributions. 


\section{Discussion}

The major possibility offered by full (3+1)D transport simulations of HQ production in HIC's concerns the study of the directed flow $v_{1}(y)$ of charm hadrons. In fact, the latter would be identically zero within a $(2+1) \mathrm{D}$ approximation assuming longitudinal boost-invariance. On the contrary its non-vanishing value allows one to obtain a full three-dimensional information on the initial state of the fireball. Furthermore, possible differences in the signal for $D^{0}$ and $\overline{D^{0}}$ mesons (requiring a better experimental investigation) must arise from the partonic stage and might be attributed to the strong initial electromagnetic fields generated from the spectator protons. Hence, coupling the transport equations to a full relativistic magneto-hydrodynamic description of the fireball - providing a consistent picture of the matter and field evolution (see e.g. [9]) - one could assess the value of the electromagnetic fields present in the medium and possibly put constraints on the electric conductivity of the QGP. We leave this issue for future work, focusing here simply on the average directed flow arising from the tilting of the fireball. Finally, the large value of the charm $v_{1}$ coefficient - much larger than the directed flow of soft particles produced at thermal equilibrium, due to the mismatch between the spatial HQ distribution and the one of the bulk matter - allows one to put tighter constraints on the value of the HQ transport coefficients: in particular the small HQ spatial diffusion coefficient $D_{s}$ prevents such an asymmetry between the distributions of heavy and soft particles to be washed out by the expansion. We refer the reader to our forthcoming publication for a more quantitative discussion on this point.

A second opportunity offered by full (3+1)D transport simulations of HQ production in HIC's concerns the possibility of providing predictions for HF measurements performed at forward rapidity. We briefly discussed this issue at the end of our contribution, referring the reader to our forthcoming publication for a more comprehensive study of the subject.

\section{References}

[1] Bozek P and Wyskiel I 2010 Phys. Rev. C 81054902 (Preprint 1002 . 4999)

[2] Adamczyk L et al. (STAR) 2014 Phys. Rev. Lett. 112162301 (Preprint 1401.3043 )

[3] Acharya S et al. (ALICE) 2019 (Preprint 1910.14406)

[4] Del Zanna L, Chandra V, Inghirami G, Rolando V, Beraudo A, De Pace A, Pagliara G, Drago A and Becattini F 2013 Eur. Phys. J. C 732524 (Preprint 1305. 7052)

[5] Adam J et al. (STAR) 2019 Phys. Rev. Lett. 123162301 (Preprint 1905. 02052)

[6] Beraudo A, De Pace A, Monteno M, Nardi M and Prino F 2015 Eur. Phys. J. C 75121 (Preprint $1410.6082)$

[7] Das S K, Plumari S, Chatterjee S, Alam J, Scardina F and Greco V 2017 Phys. Lett. B 768 260-264 (Preprint 1608.02231)

[8] Chatterjee S and Bozek P 2019 Phys. Lett. B 798134955 (Preprint 1804 . 04893)

[9] Inghirami G, Del Zanna L, Beraudo A, Moghaddam M H, Becattini F and Bleicher M 2016 Eur. Phys. J. C 76659 (Preprint 1609.03042 ) 